\title{
Una mirada sobre conceptos del cálculo desde el conocimiento de los temas del profesorado de matemática de secundaria
}

\author{
An insight into calculus concepts from the knowledge of the topics \\ of secondary education mathematics teachers
}

\section{Um olhar sobre os conceitos do cálculo desde o conhecimento dos temas dos docentes de matemática de ensino médio}

\author{
Cristián Bustos Tiemann \\ Pontificia Universidad Católica de Valparaíso \\ Valparaíso, Chile \\ cristian.bustos.t@mail.pucv.cl \\ (D) ORCID: https://orcid.org/0000-0001-8400-0662 \\ Elisabeth Ramos Rodríguez \\ Pontificia Universidad Católica de Valparaíso \\ Valparaíso, Chile \\ elisabeth.ramos@pucv.cl \\ (D) ORCID: https://orcid.org/0000-0002-8409-4125
}

Recibido - Received - Recebido: 14 / 09 / 2021 Corregido - Revised - Revisado: 29 / 10 / 2021 Aceptado - Accepted - Aprovado: 12 / 11 / 2021

DOI: https://doi.org/10.22458/ie.v24i36.3893

URL: https://revistas.uned.ac.cr/index.php/innovaciones/article/view/3893

\begin{abstract}
Resumen: Las dificultades relativas a la enseñanza y aprendizaje del cálculo implican tanto al estudiantado que cursa la asignatura como al profesorado que la imparte, más aún cuando los cambios curriculares incitan al profesorado a enseñarla en el nivel de secundaria. En este sentido, la presente comunicación tiene por objeto indagar en el conocimiento de los conceptos del cálculo: límite, derivada e integral que manifiestan las personas docentes chilenos de educación secundaria. Para ello, se ha empleado el modelo analítico del conocimiento especializado del docente de matemática, conocido como MTSK. Mediante una metodología cualitativa, se realizó un test a docentes de matemática de Chile, abordando su análisis desde la categoría definiciones, propiedades y sus fundamentos del subdominio del conocimiento de los temas. Los principales resultados dan cuenta de una tendencia a la enseñanza tradicional con énfasis en la definición erudita, con algunos aportes de la geometría dinámica para la visualización conceptual. Con este estudio, se pretende aportar a la comprensión del conocimiento del profesorado de matemática, evidenciando la manera en que se enfrentan a la enseñanza del cálculo en secundaria, en el nuevo escenario curricular en Chile y al mismo tiempo, promueva la reflexión no solo sobre el conocimiento que deben poner en juego para enseñar los objetos matemáticos límite, derivada e integral, sino que también sobre el rol que tiene cada uno de ellos en el tratamiento de los temas del cálculo para la enseñanza secundaria.
\end{abstract}

Palabras clave: MTSK, enseñanza de la matemática, cálculo, docente de secundaria, epistemología.

\begin{abstract}
Difficulties related to calculus teaching and learning involve both the students taking class and the teachers in charge, particularly when curricular changes force teachers to teach calculus at high school level In that sense, this work's objective was to inquire what teachers know about the calculus concepts of limit, derivative, and integral. In order to do that, we used the analytical model of Mathematics Teacher's Specialized Knowledge, known as MTSK. Using a qualitative methodology, we applied a test to Math teachers in Chile, addressing their analysis of the category definition, properties, and foundations of the subdomain of knowledge on the subject. The primary outcomes show the tendency to teach these topics using a traditional approach, emphasizing scholarly definition and some dynamic geometry contributions for conceptual visualization. This study intends to contribute to the understanding of mathematics teachers' knowledge, evidencing how they face the teaching of calculus in secondary school in the new curricular scenario in Chile. Moreover, it promotes reflection not only on the knowledge that they need to teach the mathematical limit, derivative and integral objects but also about their role in treating calculus issues for secondary education.
\end{abstract}

Keywords: Mathematics teaching, calculus, high school teacher, epistemology. 
Resumo: As dificuldades relacionadas ao ensino e a aprendizagem do cálculo envolvem tanto aos discentes que o estudam quanto aos docentes que o ensinam, mais ainda quando as mudanças curriculares instigam aos professores a ensinar cálculo no ensino médio. Nesse sentido, este trabalho tem por objetivo sondar o conhecimento que os docentes chilenos de ensino médio têm sobre os conceitos de cálculo: limite, derivada e integral. Para isso, empregou-se o modelo analítico do conhecimento especializado de professores de matemática, conhecido como MTSK. Mediante uma metodologia qualitativa, foi feito um teste aos professores de matemática do Chile, abordando a análises que eles tinham desde a categoria das definições, propriedades e seus fundamentos do subdomínio do conhecimento dos temas. Os principais resultados mostram uma tendência ao ensino tradicional com uma ênfase na definição erudita com algumas contribuições da geometria dinâmica para a visualização conceitual. Pretende-se com este estudo, contribuir para uma compreensão do conhecimento dos professores de matemática evidenciando a maneira na qual enfrentam o ensino do cálculo no ensino médio no novo cenário curricular no Chile e, ao mesmo tempo promovendo a reflexão não só sobre o conhecimento com o qual devem ensinar os objetos matemáticos: limite, derivada e integral, senão que também sobre o papel que tem cada um deles, no tratamento dos temas de cálculo no ensino médio.

Palavras-chave: MTSK, ensino das matemáticas, cálculo, docentes de ensino médio, epistemologia.

\section{INTRODUCCIÓN}

Si bien las problemáticas del cálculo hicieron sus primeras manifestaciones en la época de la antigua Grecia clásica, no fue sino hasta fines del siglo XVII y comienzos del siglo XVIII, en que se desarrollaron métodos sistemáticos para su resolución. En toda esa trayectoria, es posible observar lo complejo que significó para el ser humano trabajar con lo que hoy son los objetos matemáticos de límite, derivada e integral. Actualmente, las dificultades relativas a la enseñanza y aprendizaje del cálculo implican tanto a los estudiantes que cursan la asignatura como al profesorado que la imparte. Evidencia de esto se puede encontrar en numerosas investigaciones (Contreras, Luque y Ordoñez, 2003; Sánchez-Matamoros, García y Llinares, 2008), en donde destacan que, aunque se planteen cambios en su enseñanza, en general, esta se sigue impartiendo de manera tradicional.

Algunos autores (Salinas y Alanís, 2009) consideran que este paradigma tradicional, está en la secuencia de enseñanza en la que la persona docente se limita a exhibir una estructura que da cuenta de que para enseñar derivadas, por ejemplo, habrá que enseñar antes límites (porque la derivada es un límite) y para enseñar límites habrá que enseñar antes funciones (porque los límites son de funciones), y para enseñar funciones habrá que enseñar antes los números reales (porque son funciones de variable real). En este mismo esquema, el estudiantado incorporará a sus conocimientos las aplicaciones de la derivada como procedimientos repetitivos del tipo: para obtener el máximo se deriva y se iguala a 0.

Otras investigaciones mencionan que en la enseñanza del cálculo se dan, en general, dos enfoques. Por un lado, aquel en donde se favorece el uso de algoritmos y procedimientos $y$, por otro, en que beneficia los enfoques formales y teóricos (Alfaro y Fonseca, 2018). Al respecto, Bravo y Cantoral (2012) plantean que la definición formal no es adecuada para el aprendizaje, pues dicha herramienta no será utilizada posteriormente en términos prácticos y en consecuencia, no se evidenciarán elementos que puedan dotarla de sentido. Es decir, parece ser que la problemática del aprendizaje del cálculo, está ligada con el estilo de enseñanza preponderante y sus dificultades se ponen de manifiesto al considerar sus conceptos básicos como objetos de enseñanza.

Por otro lado, las investigaciones sobre lo que debiera conocer una persona docente para la enseñanza de la matemática en el aula, ha dado origen al desarrollo de diferentes modelos. Desde los trabajos de Shulman (1986), con el concepto de Conocimiento Pedagógico del Contenido, muchos investigadores han desarrollado esta línea de investigación surgiendo así el "Knowledge Quartet" (KQ) (Rowland, Huckstep y Thwaites, 2005) y el "Mathematical Knowledge for Teaching" (MKT) (Ball, Thames y Phelps, 2008), por cuanto suponen una adaptación del modelo de Shulman al dominio de la matemática. Basándose en las ideas de los modelos anteriores, surge el Conocimiento Especializado del Docente de 
Matemática (MTSK, por sus siglas en inglés), desarrollado por Carrillo et al. (2013) en la Universidad de Huelva, el que se utiliza en este estudio, dada la característica del modelo en adoptar una perspectiva intrínseca para caracterizar el conocimiento del profesorado, lo cual facilita la asignación de unidades de información, al tiempo que no necesita de explicaciones en relación con otras profesiones (Carrillo, 2017). Es posible considerar, de acuerdo con Scheiner et al. (2017), tres aspectos que hacen que el conocimiento del profesorado sea especializado: el primero, es que quienes enseñan matemáticas necesitan saber conocimientos adicionales a la materia; el segundo, es que ellos necesitan conocer la materia de una manera cualitativamente diferente a otras profesiones de las matemáticas (matemáticos, físicos, ingenieros, entre otros) y a docentes de otras materias (de física, de biología, entre otros), y el tercer aspecto, es que necesitan saber cómo organizar o estructurar la materia de manera accesible para el estudiantado.

El modelo se compone de dos grandes dominios, el dominio del conocimiento matemático (MK) y el dominio del conocimiento didáctico del contenido (PCK), cada uno de los cuales considera tres subdominios (Figura 1). Una característica relevante es que estos subdominios emanan de lo que el profesorado conoce o necesita saber para enseñar, sin necesidad de recurrir a otras profesiones, evitando los problemas de superposición que afecta a otros modelos (Carrillo et al., 2018). Es importante destacar que la diferenciación en dominios y subdominios se realiza con fines analíticos, pues se asume que el conocimiento del profesorado no es compartimentado, sino que integrado (Contreras et al., 2017).

En el esquema del modelo MTSK, que se presenta a continuación, las creencias figuran en el centro para indicar, por medio de línea segmentada, su íntima relación con todos los subdominios del conocimiento del docente.

Figura 1. Modelo del conocimiento especializado del docente de matemática, MTSK

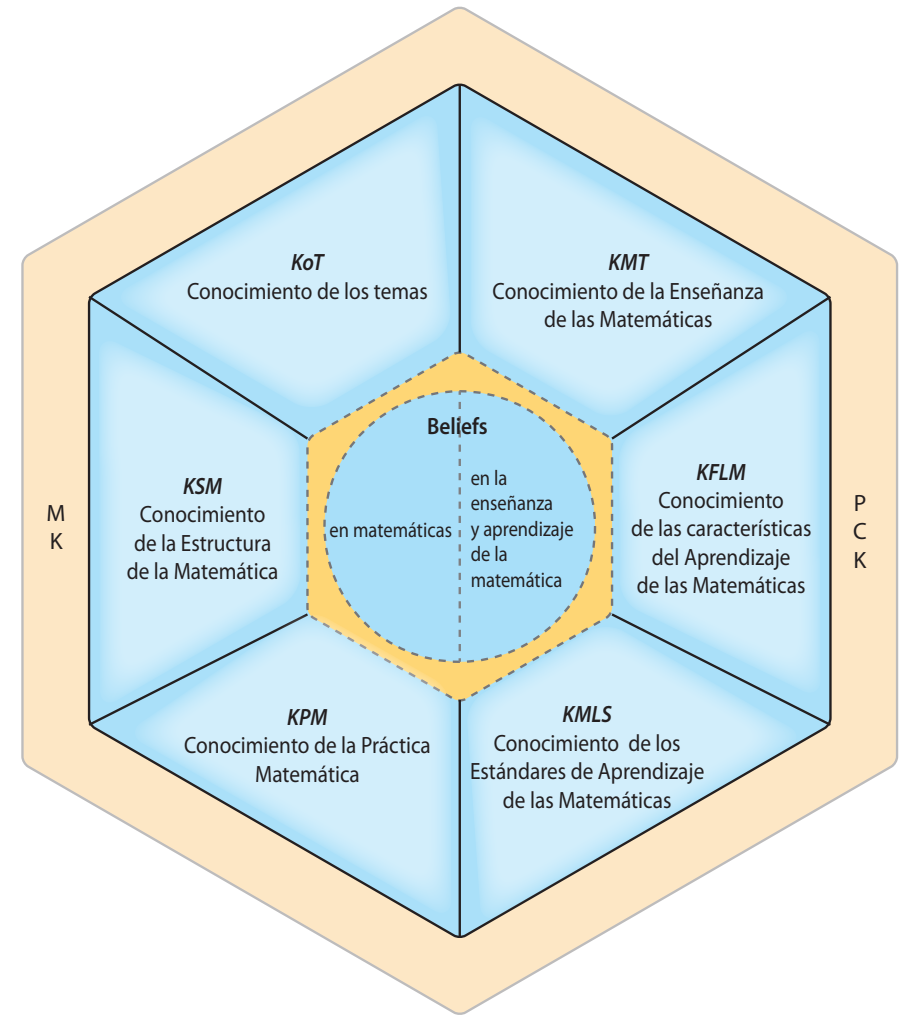

Fuente: Carrillo et al. (2018, p. 6). 
El dominio del Conocimiento Matemático (MK) se fundamenta, principalmente, en el conocimiento de la disciplina que se enseña, específicamente lo que conoce y cómo debería conocer la matemática el profesorado que enseña esta área. Si bien, el modelo contempla que la persona docente debe conocer en profundidad el contenido matemático que debe entregar (Carrillo et al., 2014; Escudero, 2015), no considera, exclusivamente. el conocimiento de la matemática como disciplina científica, sino también como un cuerpo estructurado de conocimientos que condicione su enseñanza y aprendizaje. El dominio MK está subdividido en los tres subdominios: Conocimiento de los Temas (KoT), Conocimiento de la Estructura de las Matemáticas (KSM) y el Conocimiento de la Práctica Matemática (KPM). Cada uno de estos subdominios tiene asociado sus propias categorías que permiten caracterizar a cada uno de ellos.

El dominio del Conocimiento Didáctico del Contenido (PCK) tiene como característica principal, que es un conocimiento particular del profesor, propio de la enseñanza. Este dominio también se subdivide en tres subdominios: Conocimiento de la Enseñanza de las Matemáticas (KMT), Conocimiento de las Características del Aprendizaje de las Matemáticas (KFLM) y el Conocimiento de los Estándares de Aprendizaje de las Matemáticas (KMLS). Estos subdominios se refieren, específicamente, a la práctica en aula y también tienen sus propias categorías asociadas.

Para este estudio, se ha centrado en la categoría definiciones, propiedades y sus fundamentos del KoT. El KoT del docente supone conocer el contenido matemático que se desea enseñar, junto con sus significados, propiedades y procedimientos de manera fundamentada (Carrillo et al., 2018). Por definiciones se entiende el conocimiento del profesorado sobre definiciones parciales, alternativas y las diferentes formas en que una definición puede ser explicitada, ya sea de manera oral, escrita, lenguaje natural o lenguaje matemático. Las propiedades y sus fundamentos hacen referencia al conocimiento del docente sobre las propiedades atribuidas a un tema, objeto matemático o procedimiento, que le aportan significado y sentido (Aguilar, 2016).

Sin embargo, se debe hacer notar que la categoría definiciones, propiedades y sus fundamentos se considera como un todo integrado por las dificultades en delimitar con claridad, la frontera entre definiciones y propiedades (Liñán, 2017), pues una definición involucra un conjunto de propiedades del objeto definido y, por otro lado, una propiedad puede ser usada en la definición de un tipo particular de objetos.

De acuerdo con lo señalado por Montes (2015), el subdominio KMT parece solaparse con el modelo completo, pues la especialización es entendida por el MTSK, como la integración de todos los subdominios hacia la enseñanza de la matemática. En este sentido, se consdeira la categoría estrategias, técnicas, tareas y ejemplos del KMT para este estudio. Esta categoría se enfoca en la intencionalidad de la enseñanza del profesorado, en un tema matemático.

Para el concepto de límite, se pueden apreciar diversos conocimientos del profesorado, como aquellos relacionados a la notación formal $\varepsilon-\delta$ para límites, el paso al límite como una tendencia, la aproximación intuitiva a través de la divisibilidad infinita y los límites laterales. En este sentido, existen estudios que dan cuenta de la presencia de estas ideas en el ámbito escolar y destacan, que algunas propuestas informales tienden a presentar ciertas imprecisiones, y por otra parte, aquellas con mayor formalidad tienden a ser, a veces, de un excesivo rigor y por tanto, no muy apropiadas para los estudiantes de secundaria (Blázquez, Gatica y Ortega, 2009). Otros autores (Claros et al., 2016) plantean que la introducción intuitiva del concepto de límite puede llevar a una comprensión incompleta por parte de los estudiantes y señalan que dichos enfoques deben ser complementados con sus desarrollos formales para lograr aprendizajes significativos sobre dicho concepto. Los mismos autores señalan que los enfoques intuitivos deben preceder a los enfoques formales en el desarrollo de una secuencia didáctica.

Por otro lado, para el concepto de derivada se cuenta con que el profesorado debe conocer la definición formal a través de límite, la derivada como pendiente de la recta tangente y la derivada como razón de cambio. Al respecto, se puede mencionar el estudio de García, Gavilán y Llinares (2012), en donde 
presentan la ruta del conocimiento de enseñanza para el profesorado de último año de secundaria, el cual consiste en partir por definir el concepto a través de la recta tangente a la curva, pasando por el concepto de límite para finalmente, llegar a las explicaciones basadas en los algoritmos para calcular derivadas. Se evidencia así, a través de este proceso, la forma de desarrollar su enseñanza.

En el caso del concepto de integral, destacan los conocimientos docentes sobre la integral como proceso inverso de derivar, la antiderivada, la aproximación por el límite de suma de áreas de rectángulos, la integral como área bajo la curva, la integral por medio de sumas de Riemann y las aproximaciones por medio de tablas de valores y registros gráficos. Una caracterización sobre la enseñanza de la integral la se puede ver en Alanís y Santoja (2011), que da cuenta de un énfasis en lo algorítmico desprovisto de significados, con una conceptualización basada únicamente, en la noción de área y privada de afinidad con otras ciencias, con empleo de los diferenciales por sus bondades didácticas y con la tecnología como recurso para salvar las dificultades. Dichos autores señalan, que dicha caracterización resulta incompleta para construir el proceso mismo de la integración. Por otra parte, Cordero (2005) sugiere situaciones de enseñanza que resignifiquen la integral, cuya atención se centre más en situaciones específicas de variación continua y cambio, y no directamente en los conceptos de función derivada o sumas de Riemann.

Por otro lado, en Chile, los tópicos más avanzados relacionados con el cálculo, que se trataban en la educación secundaria, tenían que ver con los conceptos de sucesión y sumatoria. Desde el año 2021 se ha incluido en el sistema escolar secundario, la asignatura de profundización "Límite, Derivadas e Integrales" con intención de abordar

Conceptos y resultados que son útiles para estudiantes de Educación Media que quieren seguir estudios superiores, técnicos o universitarios, en que la asignatura de Matemática es una herramienta central; en particular, prepara para los cursos de Cálculo que habitualmente se dictan en la Educación Superior. (Ministerio de Educación de Chile, MINEDUC, 2021, p. 21)

Este cambio curricular hace que profesores del sistema escolar chileno, se vean enfrentados a la enseñanza de temas que habían tratado en su formación inicial, pero que no habían tenido oportunidad de impartirlos en la enseñanza secundaria, lo que los lleva a retomar sus conocimientos matemáticos y didácticos sobre estos temas. De esta manera, es que este estudio se enmarca en la línea de trabajo de formación de profesores en los nuevos escenarios educativos chilenos, centrándose en el conocimiento matemático que evidencian las personas docentes de secundaria.

Bajo este contexto, surge la pregunta ¿qué conocimiento matemático de los temas (KoT) evidencian las personas docentes chilenos de secundaria sobre los conceptos del cálculo: límite, derivada e integral?, lo que lleva a plantearse como objetivo general del estudio, analizar en el conocimiento de los temas (KoT), en relación con los conceptos del cálculo: límite, derivada e integral, desde las definiciones, propiedades y sus fundamentos, que manifiestan las personas docentes chilenos de educación secundaria. Para ello, se han propuesto tres objetivos específicos: a) indagar en el conocimiento de las definiciones, propiedades y sus fundamentos de límite que manifiestan docentes chilenos, b) indagar en el conocimiento de las definiciones, propiedades y sus fundamentos de derivada que manifiestan docentes chilenos y, c) indagar en el conocimiento de las definiciones, propiedades y sus fundamentos de integral que manifiestan las personas docentes chilenos. 


\section{MATERIALES Y MÉTODOS}

Esta investigación, se desarrolla en el contexto de las nuevas bases curriculares de Chile para la enseñanza secundaria. El paradigma de investigación es de carácter cualitativo con un enfoque descriptivo-interpretativo (Hernández, Fernández y Baptista, 2014), de tal forma de describir e interpretar significados (Gibbs, 2012), los cuales son contemplados desde la perspectiva de la subdimensión KoT del MTSK en cuanto a describir, qué y de qué forma el profesorado conoce los temas.

El diseño se enmarca en un estudio de caso, considerando lo planteado por Yin (2018), en cuanto este debe permitir un acceso rápido a los datos. En este sentido, la selección de los participantes de este estudio, fue por criterio de disponibilidad y accesibilidad para responder a un test enviado por correo electrónico. Como resultado de este proceso, se cuenta con 11 sujetos informantes, los cuales son docentes de matemática de Chile, que realizan clases en la enseñanza secundaria.

El instrumento de recogida de datos, corresponde a un test (Figura 2), para cuya construcción se empleó la validación de expertos, en este caso especialistas en didáctica de la matemática, quienes revisaron el nivel de claridad y consistencia del instrumento. Para asegurar el nivel de confiabilidad del test se efectúo una prueba piloto (ensayo del instrumento), en algunos profesores de secundaria, con el fin de asegurar que la aplicación repetida del instrumento (a las mismas unidades de estudio en idénticas condiciones), produce iguales resultados.

Figura 2. Instrumento de recogida de datos

(1) En el ámbito educativo, ¿cómo definiría a sus alumnos el concepto de (límite, derivada, integral)?

(2) Entregue una actividad o tarea que haría a sus alumnos para que entienda el concepto de (límite, derivada, integral)

Fuente: Elaboración propia.

La primera pregunta apunta al conocimiento del profesorado en cuanto a las posibles definiciones, parciales, alternativas y las diferentes formas de mostrar una definición, que permitan dar sentido y significado a cada objeto matemático. Si bien tiene un enfoque orientado a cómo lo presentaría a sus estudiantes, se pretende rescatar de las respuestas, el KoT que manifiesta el docente, dado que se busca indagar en el conocimiento especializado para la enseñanza, en relación con los temas. Es decir, con ese enunciado se evita que presente su definición personal de cada tópico, sin vincularla a la enseñanza, que es lo que pretende el modelo MTSK.

En este sentido, es importante destacar que ambas preguntas, además, apuntan a la categoría estrategias, técnicas, tareas y ejemplos del KMT, lo que podría permitir evidenciar la relación entre KoT y KMT, por ejemplo, en cuanto al conocimiento de las definiciones y propiedades, pero teniendo en cuenta que en el KMT este conocimiento tiene el propósito de estrategia de enseñanza. De todas formas, esta línea de investigación se ha dejado como proyección del estudio.

Con ambas preguntas, es posible indagar en el conocimiento del profesorado sobre las propiedades que se le atribuyen a un tema, objeto matemático o procedimiento, y su relación con otros objetos junto con su fundamentación matemática. Por ejemplo, la linealidad de la derivada y la integral, cuando son consideradas como operadores, son propiedades que se pueden contemplar en esta categoría, pero que no definen al objeto matemático. Por otro lado, los registros gráficos son considerados como formas diferentes de explicitar un objeto matemático o de mostrar alguna de sus propiedades, pero sin profundizar en el conocimiento que tiene el profesorado sobre el tipo de registro, su notación o su vocabulario, por lo que la categoría registros de representación del KoT no es abordada. De igual forma, las categorías del KoT fenomenología y procedimientos, no son contempladas en este estudio, porque 
las preguntas no apuntan directamente a la aplicabilidad del objeto a situaciones fuera de un contexto matemático, en el caso de la fenomenología, y no dirigen la atención sobre el uso de algoritmos convencionales y alternativos, en el caso de los procedimientos.

Se empleó el análisis de contenido (Flick, 2004), en donde las unidades de análisis fueron las respuestas del test de cada encuestado. Las categorías de análisis surgen a partir del indicador definiciones, propiedades y sus fundamentos del KoT, considerado para este estudio, con énfasis en las definiciones tanto formales como alternativas que se han detectado en la literatura y presentadas en la sección anterior, y que se desglosa en la Tabla 1.

TABLA 1

Categorías de análisis

\begin{tabular}{l|l}
\multicolumn{1}{c}{ Concepto } & \multicolumn{1}{c}{ Categoría } \\
\hline \multirow{2}{*}{ Límite } & La notación formal $\varepsilon$ - $\delta$ para límites \\
\hline & El paso al límite como una tendencia \\
\hline & Aproximación intuitiva a través de la divisibilidad infinita \\
\hline & Aproximación por medio de tablas de valores \\
\hline \multirow{5}{*}{ Uerivada } & Exo de registro gráfico por medio de la geometría (estática o dinámica) \\
\hline Definición formal de la derivada a través de límite \\
\hline & La derivada como pendiente de la recta tangente \\
\hline La derivada como razón de cambio \\
\hline Uso de registro gráfico por medio de la geometría (estática o dinámica) \\
\hline La integral como proceso inverso de derivar, la antiderivada \\
\hline & Aproximación por el límite de suma de áreas de rectángulos \\
\hline & La integral como área bajo la curva \\
\hline La integral por medio de sumas de Riemann \\
\hline
\end{tabular}

El análisis de datos se ha enfocado en las respuestas presentadas para ambas preguntas (unidades de análisis), codificando globalmente ambas respuestas en qué categoría se ubican, teniendo en cuenta que algunas de ellas se pueden situar en más de una categoría. Posterior a ello, y en concordancia con los tres objetivos específicos propuestos, se observa de manera integrada los hallazgos, en relación con el conocimiento de las definiciones, propiedades y sus fundamentos en cada tópico abordado, es decir, para límite, luego para derivada y finalmente, la integral.

\section{DISCUSIÓN DE RESULTADOS}

Los principales resultados dieron cuenta de una tendencia del profesorado hacia un conocimiento formal, para explicitar los conceptos de límite, derivada e integral. Al organizar los datos recogidos se obtuvo la Tabla 2, que muestra las categorías y sus frecuencias surgidas de las producciones del profesorado. 
TABLA 2

Resumen de la presencia de las categorías en las respuestas de los profesores

\begin{tabular}{|c|c|c|}
\hline Límite & Derivada & Integral \\
\hline Definición formal de límite de función 2/11 & $\begin{array}{l}\text { Definición formal de la derivada a } \\
\text { través de límite } \mathbf{2 / 1 1}\end{array}$ & Antiderivada 2/11 \\
\hline Como tendencia o aproximación 4/11 & Razón de cambio 2/11 & Área bajo la curva 5/11 \\
\hline Existencia de límites laterales 2/11 & Pendiente de recta tangente $\mathbf{8 / 1 1}$ & Sumas de Riemann $\mathbf{2 / 1 1}$ \\
\hline Definición formal de límite de sucesión 1/11 & No responde $\mathbf{2 / 1 1}$ & $\begin{array}{l}\text { Aproximación por el límite de suma } \\
\text { de áreas de rectángulos } 6 / 11\end{array}$ \\
\hline Divisibilidad infinita 2/11 & Uso de registro gráfico 8/11 & No responde $\mathbf{2 / 1 1}$ \\
\hline Uso de registro gráfico 3/11 & & Uso de registro gráfico 5/11 \\
\hline
\end{tabular}

Fuente: Elaboración propia.

En muchos casos, se dio más de una categoría para cada objeto matemático, pues hay docentes que definieron, por ejemplo, la derivada como la pendiente de la recta tangente a la curva en un punto y además, como una razón de cambio. Por otro lado, algunos de ellos, que declararon que preferían comenzar por proponer la actividad o tarea y después entregar una definición que surgiera de la misma. Los dos docentes que no respondieron en derivada e integral, resultaron ser los mismos.

A continuación, se muestra el análisis de los hallazgos, en relación con cada objetivo específico del estudio; es decir, iniciando con un estudio en relación con el concepto de límite, luego de derivada y finalmente, de la integral.

\section{Resultados para el concepto de límite}

Para el concepto de límite se dieron tres casos, donde el profesorado lo definió usando la notación formal $\varepsilon-\delta$, dos para funciones y uno para sucesión, dando cuenta de una definición más cercana al saber sabio en el nivel de secundaria. En la Figura 3, se puede ver un ejemplo, donde se manifiesta conocimiento del docente sobre el concepto de límite desde la definición formal para funciones.

Figura 3. Respuesta que evidencia conocimiento del docente sobre la definición formal de límite

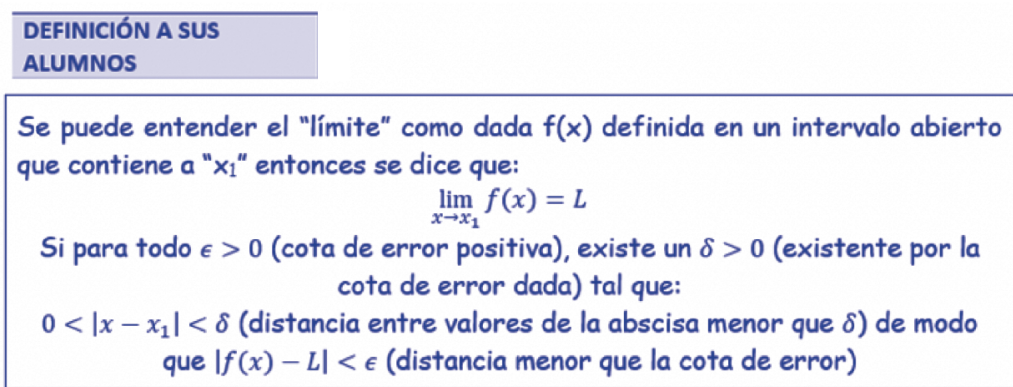

Fuente: Elaboración propia.

También, se destaca el obstáculo epistemológico de considerar el paso al límite, como una tendencia o aproximación. Se pueden ver dos ejemplos de ello en la Figura 4. 
Figura 4. Respuestas que manifiestan conocimiento del docente sobre límite como tendencia o aproximación

\begin{tabular}{|l|}
\hline \begin{tabular}{l}
\hline DEFINICIÓN A SUS \\
ALUMNOS
\end{tabular} \\
\hline $\begin{array}{l}\text { Como el concepto } \\
\text { que describe la } \\
\text { tendencia que } \\
\text { tiende una sucesión } \\
\text { o función. } \\
\text { También mediante } \\
\text { algunos gráficos. }\end{array}$ \\
\hline
\end{tabular}

\begin{tabular}{|l|}
\hline \\
\hline DEFINICIÓN A SUS \\
ALUMNOS \\
\hline Es un estudio de \\
tendencia hacia \\
cierto lugar \\
dentro de una \\
función, es decir, \\
ver que sucede \\
cuando uno se \\
acerca mucho \\
hacia algún sitio \\
que, por lo \\
general, es \\
controversial. \\
\hline
\end{tabular}

Fuente: Elaboración propia.

Se dan dos casos que evidenciaron el conocimiento del concepto de límite, desde un enfoque intuitivo a través de la divisibilidad infinita, lo que está más acorde al saber, a enseñar lo planteado en el programa del MINEDUC (2021). Se ve esta situación en la Figura 5.

Figura 5. Respuesta que manifiesta conocimiento del docente sobre límite desde un enfoque intuitivo

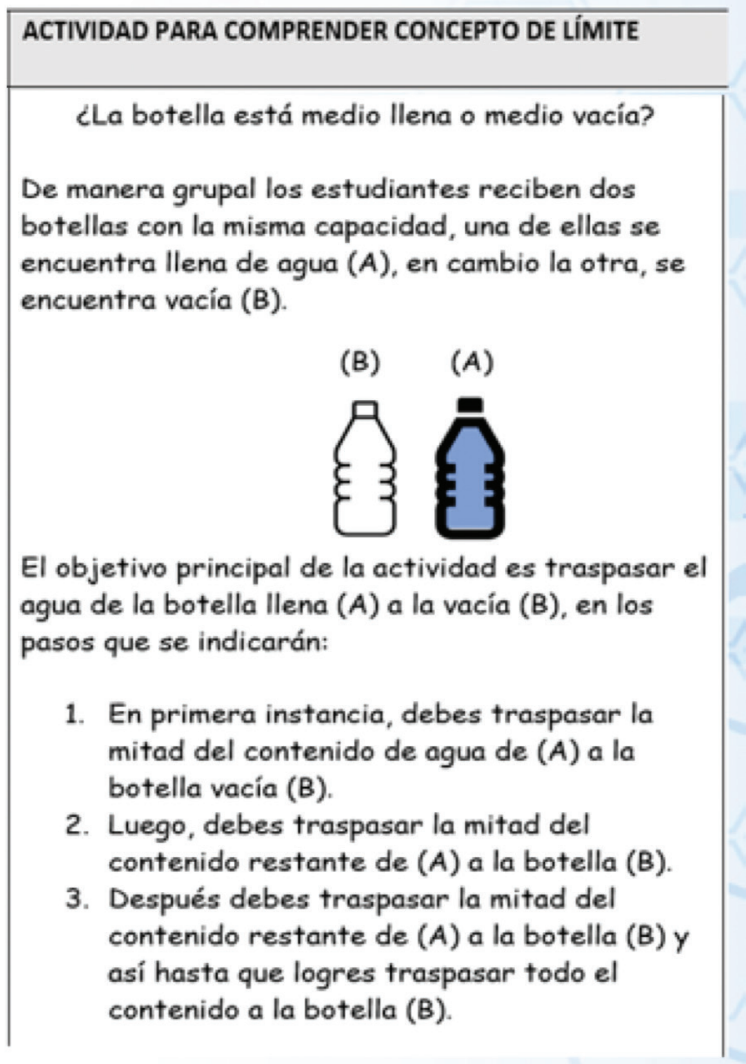

Fuente: Elaboración propia. 
Para los límites laterales, el profesorado consideró actividades de completar tablas, acercándose por valores desde la derecha y desde la izquierda, como se ilustra en la Figura 6, donde manifestaron su conocimiento de límite desde la existencia de límites laterales.

Figura 6. Respuesta que manifiesta conocimiento del docente sobre límite a través de la existencia de límites laterales

\section{ACTIVIDAD PARA COMPRENDER CONCEPTO DE LÍMITE}

1. Complete la tabla de valores utilizando 4 decimales y estime el valor del límite.

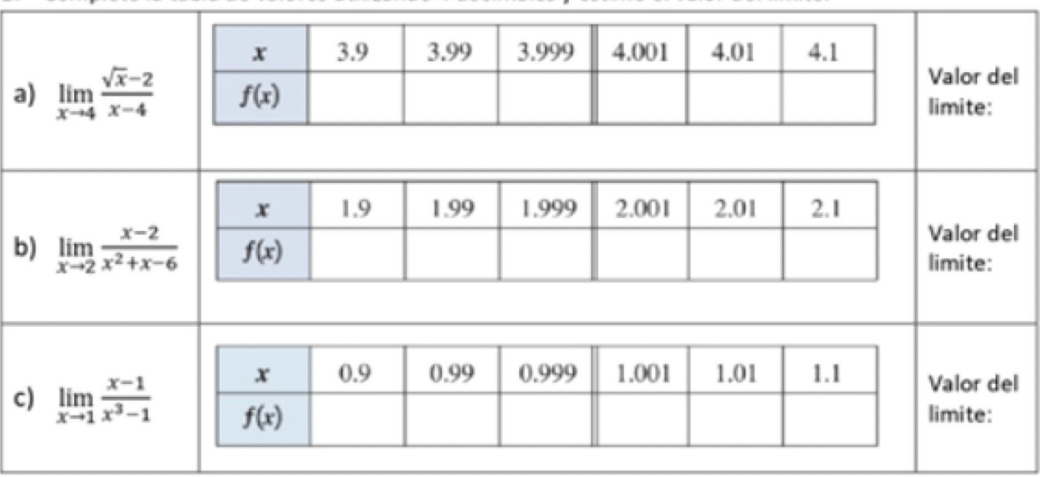

Fuente: Elaboración propia.

También se evidenció conocimiento de tres docentes, en relación con el límite desde el análisis de las gráficas de algunas funciones, para visualizar diferentes formas de acercamiento. Se puede ver, de los tres ejemplos, evidencia de este conocimiento en la Figura 7. Sin embargo, de los 11 sujetos encuestados, ninguno mencionó el uso de GeoGebra, en tanto que sí lo hicieron algunos con los conceptos de derivada e integral, como se verá más adelante.

Figura 7. Respuestas que manifiestan conocimiento del docente Sobre el límite a través del análisis de gráficos

\begin{tabular}{|c|c|c|}
\hline & TIVIDAD PARA COMPRENDER CONCEPTO DE LÍMITE & \\
\hline $\begin{array}{l}\text { Si o si, haría la enseñanza de límite con la } \\
\text { representación gráfica acerca de lo que necesito } \\
\text { que interpreten (gráfica del límite), de cierta } \\
\text { manera, es para darle sentido a la definición } \\
\text { teóricol }\end{array}$ & $\begin{array}{l}\text { Analizar gráficamente diversos puntos } f(x) \text { por izquierda y } \\
\text { derecha, como autos por rutas acercándose por ambos } \\
\text { lados y verificar si estos chocan o no. }\end{array}$ & $\begin{array}{l}\text { Analizar, mediante la realización de gráficos, el } \\
\text { concepto de limite. } \\
\text { - Grafique un esquema superficial de la } \\
\text { función } f(x)=\log _{a} x \text {, y analice que sucede } \\
\text { cuando ésta va creciendo. }\end{array}$ \\
\hline Ejemplo 1 & Ejemplo 2 & Ejemplo 3 \\
\hline
\end{tabular}

Fuente: Elaboración propia.

\section{Resultados para el concepto de derivada}

Respecto del objeto derivada, solo dos docentes manifestaron conocimiento para su enseñanza a través de la definición formal como un límite, pero después en las actividades, solicitaron encontrar la pendiente de la recta tangente en un punto, sin explicitar cómo relacionan la definición dada con la interpretación geométrica. Esto se puede evidenciar en la Figura 8, en donde se pretende que el alumno visualice la pendiente de la recta tangente a partir de la definición formal. 
Figura 8. Respuesta que evidencia conocimiento del docente sobre la definición formal de derivada y conocimiento de la derivada como pendiente de recta tangente

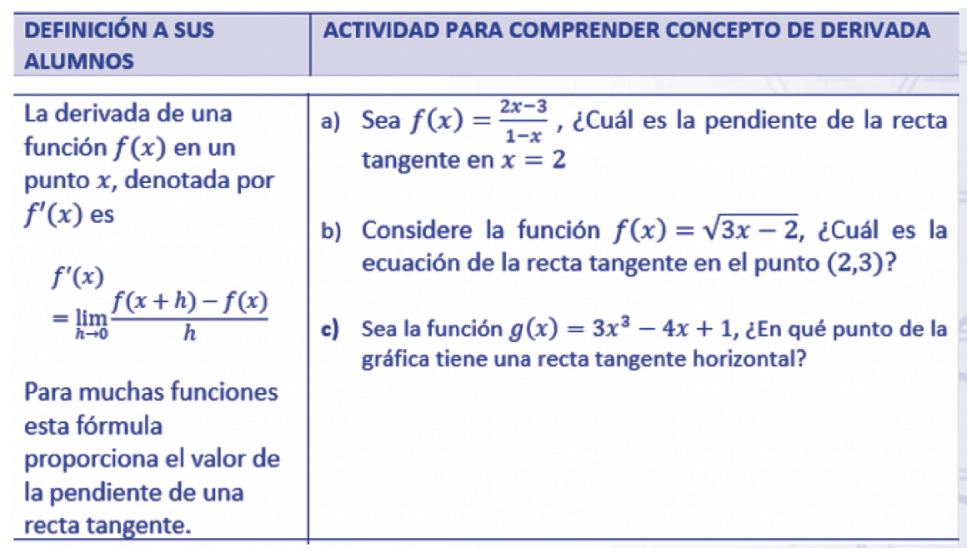

Fuente: Elaboración propia.

La mayoría del profesorado (8 de 11) explicó el concepto de derivada como la pendiente de la recta tangente por medio del análisis de gráficos de funciones, usando GeoGebra, es decir, por medio de una actividad gráfica y no con la definición formal. Ejemplo de esto se puede ver en la Figura 9, en donde se evidencia conocimiento del docente sobre la enseñanza de la derivada como pendiente de la recta tangente, por medio de una actividad con GeoGebra.

Figura 9. Respuesta que evidencia conocimiento del docente sobre la pendiente asociada a límite por medio de la geometría dinámica

\begin{tabular}{l|l|}
\hline $\begin{array}{l}\text { DEFINICIÓN A SUS } \\
\text { ALUMNOS }\end{array}$ & ACTIVIDAD PARA COMPRENDER CONCEPTO DE DERIVADA \\
\hline $\begin{array}{l}\text { Al igual que en el } \\
\text { concepto de limites, el } \\
\text { concepto de derivada lo } \\
\text { introduzco con una } \\
\text { actividad (en este caso } \\
\text { un GeoGebra), de tal } \\
\text { manera que los puntos, y verificar qué pasa cuando esos puntos son } \\
\text { "coincidentes". } \\
\text { estudiantes asocien el } \\
\text { concepto de limites con } \\
\text { la pendiente, dos } \\
\text { conceptos trabajados } \\
\text { previamente. }\end{array}$ &
\end{tabular}

Fuente: Elaboración propia.

En una de las respuestas entregadas, se planteó analizar gráficos de distancia versus tiempo, en donde la derivada representa la velocidad, a pesar de no mostrar una actividad que acompañe esta idea. Sin embargo, esto es más acorde con el programa de MINEDUC (2021), en donde se describe la derivada como función de pendientes de rectas tangentes, después de considerarla como razón de cambio con ejemplos asociados a la velocidad instantánea. Además, esta idea de la derivada asociada a la pendiente, corresponde al saber a enseñar y responde a uno de los problemas que dieron origen a la derivada (Ávila et al., 2013). 


\section{Resultados para el concepto de integral}

En cuanto al concepto integral, en general se dieron dos tipos de propuestas de enseñanza, la integral como antiderivada y la integral como área bajo la curva. En el caso de la integral como antiderivada se pide, además, calcular la primitiva de algunas funciones, como se puede observar en la Figura 10, en donde se manifiesta conocimiento del docente sobre la integración como cálculo de primitivas.

Figura 10. Respuesta que evidencia conocimiento del docente sobre la integración como antiderivación y cálculo de primitivas

\begin{tabular}{|c|c|}
\hline DEFINICIÓN A SUS ALUMNOS & $\begin{array}{l}\text { ACTIVIDAD PARA COMPRENDER CONCEPTO DE } \\
\text { INTEGRAL }\end{array}$ \\
\hline & \\
\hline 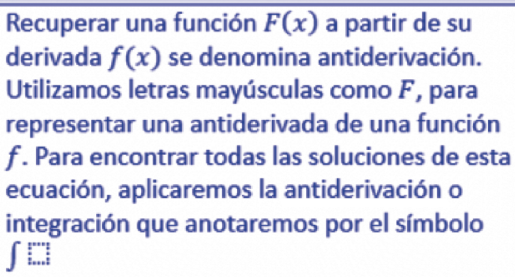 & $\begin{array}{l}\text { Calcula la función primitiva (anti-derivada) de } \\
\text { las funciones: } \\
\text { a) } g(x)=7 x^{3}-6 x \\
\text { b) } h(x)=\sqrt[5]{x^{2}}+\frac{3}{x^{5}}\end{array}$ \\
\hline \multicolumn{2}{|l|}{ Entonces la solución general será: } \\
\hline $\int(x) d x=F(x)+C$ & \\
\hline
\end{tabular}

Fuente: Elaboración propia.

Sin embargo, en la propuesta de integral como área bajo la curva, se distinguen dos miradas. Una, donde el profesor manifestó conocimiento sobre las sumas de Riemann, como se ve en la Figura 11, en la cual se observa el uso de dicha concepción para su enseñanza.

Figura 11. Respuesta que evidencia conocimiento docente sobre la integral a través de sumas de Riemann y el cálculo de áreas

\begin{tabular}{l|l}
\hline DEFINICIÓN A SUS ALUMNOS & $\begin{array}{l}\text { ACTIVIDAD PARA COMPRENDER CONCEPTO DE } \\
\text { INTEGRAL }\end{array}$ \\
\hline \multicolumn{2}{|l}{} \\
\hline $\begin{array}{l}\text { A través de sus aplicaciones, específicamente } \\
\text { a través del cálculo de área y la suma de }\end{array}$ & $\begin{array}{l}\text { Calcule el área de figuras que no sean } \\
\text { poligonos, como por ejemplo el área bajo } \\
\text { una curva }\end{array}$ \\
\hline
\end{tabular}

Fuente: Elaboración propia.

La segunda propuesta, ilustrada a través de dos ejemplos en la Figura 12, en donde se manifestó un conocimiento que emerge de manera más intuitiva, usando el método exhaustivo para calcular el área de un círculo o mediante, la suma de las áreas de rectángulos que dividan la superficie bajo la curva. 
Figura 12. Respuestas que evidencian conocimiento docente de la integral como área bajo la curva

\section{ACTIVIDAD PARA COMPRENDER CONCEPTO DE INTEGRAL}

Les entregaría un círculo y les pediría calcular el área sin utilizar la fórmula conocida. Por lo que les propondria a cada alumno o alumnos inscribir figuras geométricas con $\mathrm{n}$ lados, así desarrollariamos el método del agotamiento. Luego esto lo relacionamos con la gráfica de una función $\mathrm{f}(\mathrm{x})$ en donde tenga partes de cálculo sencillo (áreas de figuras conocidas) como partes en que existan curvas donde no se pueda calcular el área tan fácilmente.
Obj: Reconocer, a través de ejemplas gráficos, el concepto de integral como área bajo la curva

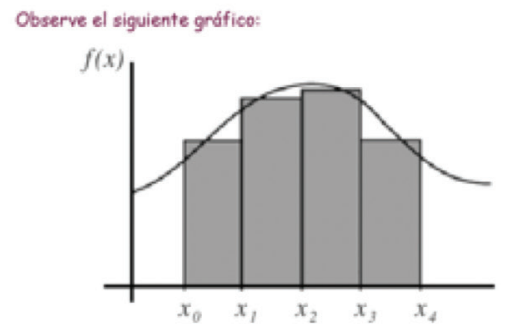

Podremos notar que bajo la curva encontraremos rectángulos, y que éstos se asemejan mucho a la curva de la función. Si calculásemos el área de cada uno de ellos y la sumásemos, este sería un primer acercomiento al órea bajo la función.

Esto lo podemos hacer con cualquier función, ya sea que tenga curvas (come por ejemplo una perobola) o sea una función lineal, come por ejemplo $\mathrm{x}=3$.

\section{Ejemplo 1}

\section{Ejemplo 2}

Fuente: Elaboración propia.

Esta segunda mirada, se corresponde con el saber a enseñar y la propuesta de MINEDUC (2021). Se presentó también el recurso del registro gráfico por medio de la geometría estática (dibujo con lápiz en papel), como se ve en la Figura 13, en donde se manifestó conocimiento del docente sobre la integral como área bajo la curva.

Figura 13. Respuesta que evidencia conocimiento del docente de la integral como área bajo la curva por medio de la geometría estática

\section{ACTIVIDAD PARA COMPRENDER CONCEPTO DE INTEGRAL}

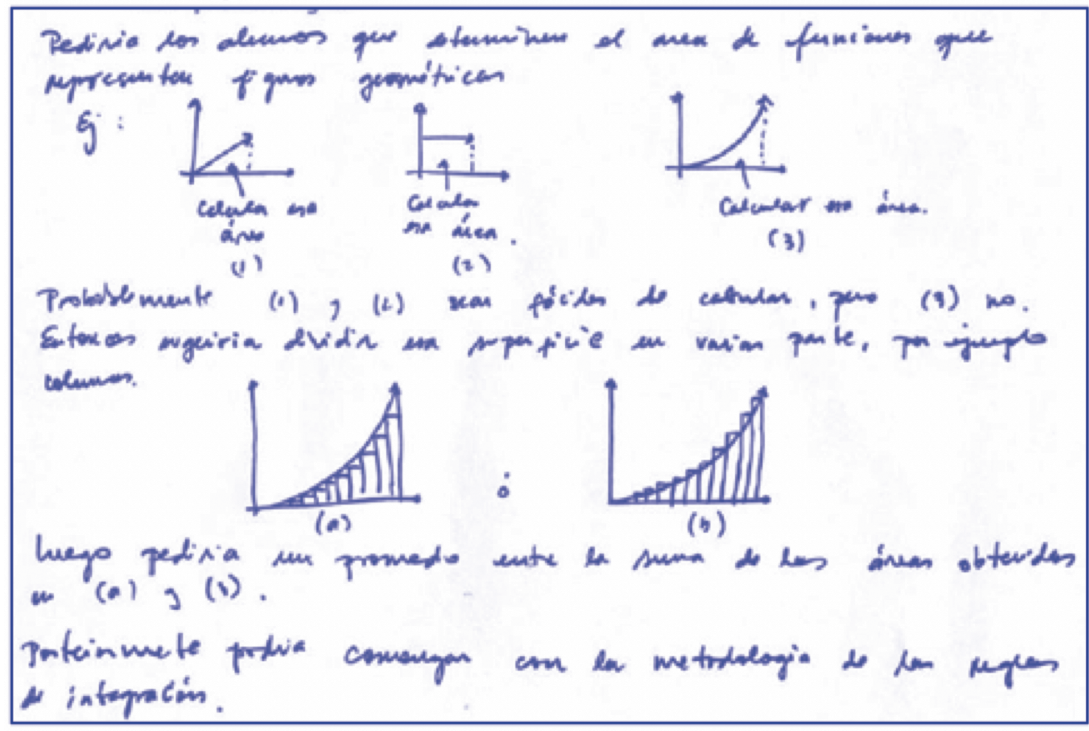

Fuente: Elaboración propia. 
Un ejemplo de uso del registro gráfico, por medio de la geometría dinámica, se puede observar en la Figura 14, en donde se manifestó el conocimiento del docente sobre estimar el área bajo la curva, usando sumas superiores e inferiores de rectángulos haciendo uso de GeoGebra.

Figura 14. Respuesta de un profesor que evidencia conocimiento del docente de la integral, como área bajo la curva por medio de la geometría dinámica

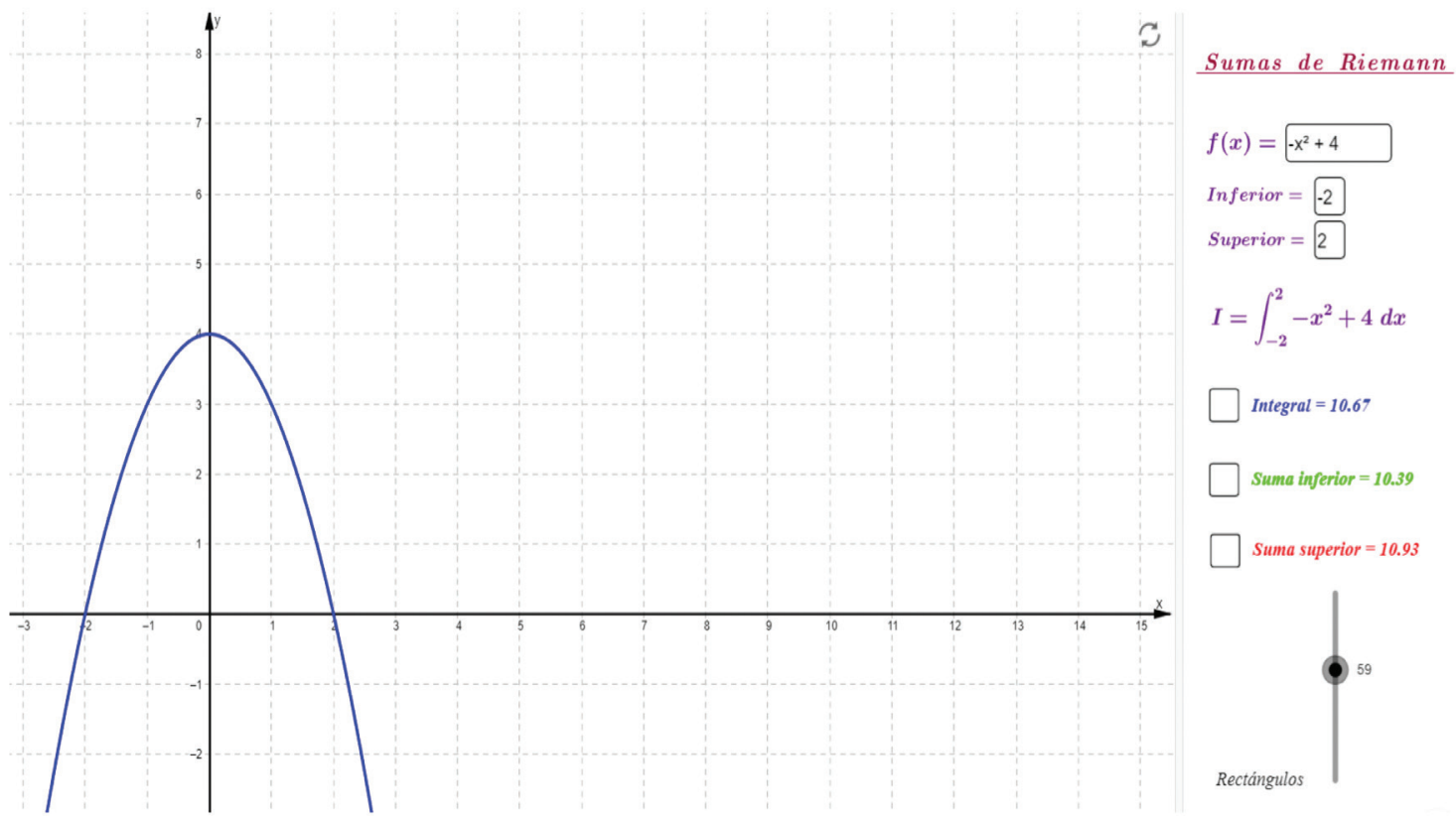

Fuente: Elaboración propia.

\section{SÍNTESIS Y REFLEXIONES FINALES}

Este estudio se ha propuesto como objetivo principal, analizar el conocimiento de los temas (KoT), en relación con los conceptos del cálculo: límite, derivada e integral, desde las definiciones, propiedades y sus fundamentos que manifiestan docentes chilenos de educación secundaria. De acuerdo con los resultados, es posible observar, en el profesorado encuestado, una tendencia a evidenciar conocimiento de los conceptos límite, derivada e integral desde una mirada formal, como se evidenció en el estudio de Alfaro y Fonseca (2018), en donde se destaca la inclinación por el enfoque formal como una de las miradas preponderantes en la enseñanza del cálculo.

Se asume, por ejemplo, la definición de derivada como el límite de un cociente diferencial o la integral, como el límite de una suma de Riemann, lo cual, según diversos autores (Alanís y Salinas, 2009; Opazo, Marcía y Cordero, 2020), no es lo más apropiado para lograr aprendizajes significativos sobre dichos objetos matemáticos, pues para alcanzar su comprensión es necesario conocer primeramente, el problema o clase de problemas que permitieron su surgimiento y evolución. Además, el profesorado presenta ejercicios encaminados al cálculo de primitivas o área bajo la curva, en el caso de las integrales o al cálculo de la pendiente de la recta tangente dadas ciertas funciones, principalmente algebraicas. Esta visión, es contraria a la manifestada por Cordero (2005), quien sugiere, como se menciona anteriormente, optar por situaciones que se enfoquen más en situaciones de variación continua y de cambio. 
En general, el profesorado manifiesta su conocimiento sobre el uso de la geometría dinámica principalmente, para visualizar la idea contenida en el concepto. La utilización de GeoGebra, y no otra herramienta digital, es también común a todos. Respecto del manejo de la tecnología, se cree oportuno destacar, de acuerdo con Artigue (2002), que no basta un buen manejo de la misma, sino que debe existir una parte epistémica que emerja de este proceso (instrumentación) y que se integre al conocimiento para comprender un contenido matemático.

Es de notar, además, que hubo cuatro docentes, de los 11 encuestados, que manifiestan no tener conocimiento sobre cómo responder a los conceptos de derivada e integral, incluso algunos declaraban que no sabrían cómo definir dichos conceptos o qué tipo de actividad podrían llevar al aula escolar (aún no lo han enseñado). Una posible explicación a esta situación puede tener su origen, en que ellos no recuerdan dichos objetos matemáticos o que perciban que su tratamiento a nivel de secundaria debe ser diferente a su formación disciplinar recibida y, en consecuencia, presenten dificultades en su conocimiento sobre cómo llevar al aula una propuesta de enseñanza. Esto pone de relieve una debilidad del estudio, en torno a la diversidad de instrumentos de recogida de datos considerados, pero que invita hacia futuras investigaciones, donde se indague sobre ello, a través de entrevistas en profundidad o se realice un seguimiento en el aula en aquellos docentes.

De acuerdo con Steele et al. (2013), al definir un objeto matemático, el profesorado debe decidir sobre varias posibilidades y seleccionar aquella que se ajuste al objetivo de su enseñanza, esto puede llevar a destacar las relaciones que pueden originarse entre el KoT y el MKT, en relación con la categoría estrategias, técnicas, tareas y ejemplos de este. Por ejemplo, cuando un profesor define un concepto (manifestando su KoT), se puede tener en cuenta cuál es el propósito de estrategia de enseñanza que está detrás de su definición (KMT). Esta relación interesante que se provoca entre ambos subdominios del MTSK, se ha dejado como proyección del estudio.

Se espera que este estudio evidencie la manera, en que docentes de matemática enfrentan la enseñanza del cálculo a la luz de las nuevas exigencias curriculares en Chile y al mismo tiempo, promueva la reflexión no solo sobre el conocimiento que deben poner en juego para enseñar los objetos límite, derivada e integral, sino que también sobre el rol que tiene cada uno de ellos, en la estructuración y tratamiento de los temas de cálculo para la enseñanza secundaria.

\section{AGRADECIMIENTOS}

Esta investigación está financiada parcialmente por la Agencia Nacional de Investigación y Desarrollo, ANID, del Gobierno de Chile, proyecto FONDECYT 11190553, y por la Vicerrectoría de Investigación y Estudios Avanzados de la Pontificia Universidad Católica de Valparaíso, Beneficiario Beca Postgrado PUCV 2021. Este trabajo está vinculado a la Red MTSK de la Asociación Universitaria Iberoamericana de Posgrado (AUIP). 


\section{REFERENCIAS}

Agullar, A. (2016). El conocimiento especializado de una maestra sobre la clasificación de las figuras planas. Un estudio de caso. Tesis doctoral. Huelva, España: Universidad de Huelva.

Alanis, J. A. у Soto, E. (2011). La integral de funciones de una variable: Enseñanza actual. Revista de Cálculo y su enseñanza, 3, 1-6.

AlFARo, C. y FonseCA, J. (2018). Problem solving in the teaching of differential and integral calculus in one variable: Perspective of mathematics teachers. Revista Uniciencia. 32(2), 42-56.

ARTIGUE, M. (2002). Learning mathematics in a CAS environment: The Genesis of a Reflection about instrumentation and the Dialectics between Technical and Conceptual Work. International Journal of computers for Mathematical Learning, 7(3), 245-274.

ÁvILA, J.; ÁviLA, R. y ParRA, F. (2013). Desarrollo histórico-epistemológico de la derivada de una función. En R. Flores (Ed.), Acta Latinoamericana de Matemática Educativa Volumen 26 (pp. 1223-1230). México, DF: Comité Latinoamericano de Matemática Educativa.

Ball, D. L.; Thames, M. H. y Phelps, G. (2008). Content knowledge for teaching: What makes it special? Journal of Teacher Education, 59(5), 389-407.

Blázquez, S.; Ortega, T.; Gatica, S. y Benegas, J. (2006). Una conceptualización de límite para el aprendizaje inicial de análisis matemático en la universidad. Revista latinoamericana de investigación en matemática educativa, 9(2), 189-209.

Bravo, S. y CAntoral, R. (2012). Los libros de texto de Cálculo y el fenómeno de la Transposición Didáctica. Revista Educación Matemática, 1(1), 20-22.

Carrillo, J.; Climent, N.; Contreras, L. C. y Muñoz-Catalan, M. C. (2013). Determining specialized knowledge for mathematics teaching. En B. Ubuz, C. Hasery M. Mariotti (Eds.), Proceedings of the CERME 8 (pp. 2985-2994). Antalya, Turquía: CERME.

CARrillo, J.; Escudero, D. y Flores, E. (2014). El uso del MTSK en la formación inicial de profesores de matemáticas de primaria. FOR-MATE. Revista de Análisis Matemático-Didáctico para profesores, 1(1), 16-26.

CARRILLo, J. (2017). Idiosincrasia del MTSK, Investigaciones realizadas y utilidades. En J. Carrillo y L. C. Contreras (Eds.), Avances, utilidades y retos del modelo MTSK. Actas de las III Jornadas del Seminario de investigación de Didáctica de la Matemática de la Universidad de Huelva (pp. 7-10). Huelva: CGSE.

Carrillo, J.; Climent, N.; Montes, M.; Contreras, L.; Flores-Medrano, E.; Escudero-Ávila, D.; Vasco, D.; Rojas, N.; Flores, P.; Agullar-Gonzalez, A.; Ribelro, M. y Muñoz-Catalán, C. (2018). The mathematics teacher's specialized knowledge (MTSK) model. Research in Mathematics Education, 20(3), 236-253.

Claros, J.; Sánchez, M. y Coriat, M. (2016). Tratamiento del límite finito en libros de texto españoles de secundaria: 1933-2005. Educación Matemática 28(1).

Contreras, A.; Luque, L. y Ordoñez, L. (2003). Una perspectiva de la enseñanza aprendizaje de la continuidad y la derivada de una función en bachillerato y universidad. Revista de Educación, 331, 399-419.

Contreras, L. C.; Montes, M.; Climent, N. y Carrillo, J. (2017). Introducción al modelo MTSK: origen e investigaciones realizadas. Revista For-Mate, 3, 7-15.

CoRDERO, F. (2005). El rol de algunas categorías del conocimiento matemático en educación superior. Una Socioepistemología de la integral. Revista Latinoamericana de Investigación en Matemática Educativa, 8(3), 265-286.

EsCUDERO, D. (2015). Una caracterización del conocimiento didáctico del contenido como parte del conocimiento especializado del profesor de matemática de secundaria. Tesis doctoral. Huelva, España: Universidad de Huelva. 
FLICK, U. (2004). Introducción a la Investigación Cualitativa. Ediciones Morata S.L.

Garcia, M.; GaVILAN, J. M. y Llinares, S. (2012). Perspectiva de la práctica del profesor de matemáticas de secundaria sobre la enseñanza de la derivada. Relaciones entre la práctica y la perspectiva del profesor. Enseñanza de las Ciencias, 30(3), 219-235.

GIBBS, G. (2012). El análisis de datos en investigación cualitativa. Madrid: Ediciones Morata.

Hernandez, R.; Fernández, C. y Baptista, M. (2014). Metodología de la investigación (6ta. edición). Editorial McGraw-Hill.

LIÑAN, M. M. (2017). Conocimiento especializado en geometría en un aula de $5^{\circ}$ de primaria. Tesis doctoral. Huelva, España: Universidad de Huelva.

Ministerio de Educación de Chile [MINEDUC]. (2021). Matemática. Programa de Estudio Límites, Derivadas e Integrales para Formación Diferenciada $3^{\circ}$ y $4^{\circ}$ Medio. Santiago de Chile: Ministerio de Educación.

Montes, M. (2015). Conocimiento especializado del profesor de matemáticas acerca del infinito. Un estudio de caso. Tesis doctoral. Huelva, España: Universidad de Huelva.

Opazo, A.; Marcia, S. y Cordero, F. (2020). Adherencia al discurso Matemático Escolar: el caso de la integral definida en la formación inicial docente. UCMaule, 59, 31-55.

Rowland, T.; Huckstep, P. y Thwaites, A. (2005). Elementary teachers' mathematics subject knowledge: The knowledge quartet and the case of Naomi. Journal of Mathematics Teacher Education, 8(3), 255-281. doi:10.1007/s10857-005-0853-5

Salinas, P. y Alanis, J. (2009). Hacia un nuevo paradigma en la Enseñanza del cálculo dentro de una institución educativa. Revista Latinoamericana de Investigación en Matemática Educativa 12(3), 355-382.

Sánchez-Matamoros, G.; García, M.; y Llinares, S. (2008). La comprensión de la derivada como objeto de investigación en didáctica de la matemática. Revista Latinoamericana de Investigación en Matemática Educativa, 11(2), 267-296.

Scheiner, T.; Montes, M. A.; Godino, J. D.; Carrillo, J. y Pino-Fan, L. (2017). What Makes Mathematics Teacher Knowledge Specialized? Offering Alternative Views. International Journal of Science and Mathematics Education 17(1), 153-172. https://doi.org/10.1007/s10763-017-9859-6

SHULmAN, L. (1986). Those who understand. Knowledge growth in teaching. Educational Researcher, 15(2), 4-14.

YIN (2018). Case study research and applications: Design and methods. Thousand Oaks: SAGE Publications, Inc. 